\title{
Can rationing possibly be rational?
}

A nnie Farlow was just short of three months old when she died in an Ontario hospital of what her parents believe might have been a treatable respiratory condition.

Born with the chromosome disorder Trisomy 13, Annie had little chance of surviving to her teens, but her parents, Barbara and Tim Farlow, took comfort in the hospital's assurance that her disorder wouldn't preclude her from receiving the same level of care as any other child with a medical condition.

But months after Annie's death, the Farlows discovered that physicians had not initiated emergency resuscitation measures with the same alacrity as in other cases. They also learned that a physician had placed a "do not resuscitate" order on Annie's chart without their consent. Subsequently, their daughter received an undisclosed quantity of narcotics that, in their opinion, may have caused her "unexplained, rapid" decline.

The circumstances surrounding Annie's death have the Farlows asking questions that plague Canadian patient advocates, health care workers, ethicists and policy-makers faced with a growing scarcity of resources: When is it appropriate to limit or withdraw potentially beneficial treatment? How should decisions be made? Who should make them?

"I never took the position that my daughter had a right to any and all treatments, but the unilateral decisions we believe the doctors made should have been made transparently. We have a right to know and appeal the limits of the system and be provided with whatever care is possible within its confines," Barbara Farlow says. "Not only do I believe my daughter was denied a chance to prolong her life, the secrecy in which decisions seem to have been made also meant she was denied timely palliative care, and she suffered greatly at the end."

Farlow has since joined other Canadians in calling for an "open, honest and public" conversation about health care rationing in hope of addressing hidden bias in current decision-making processes

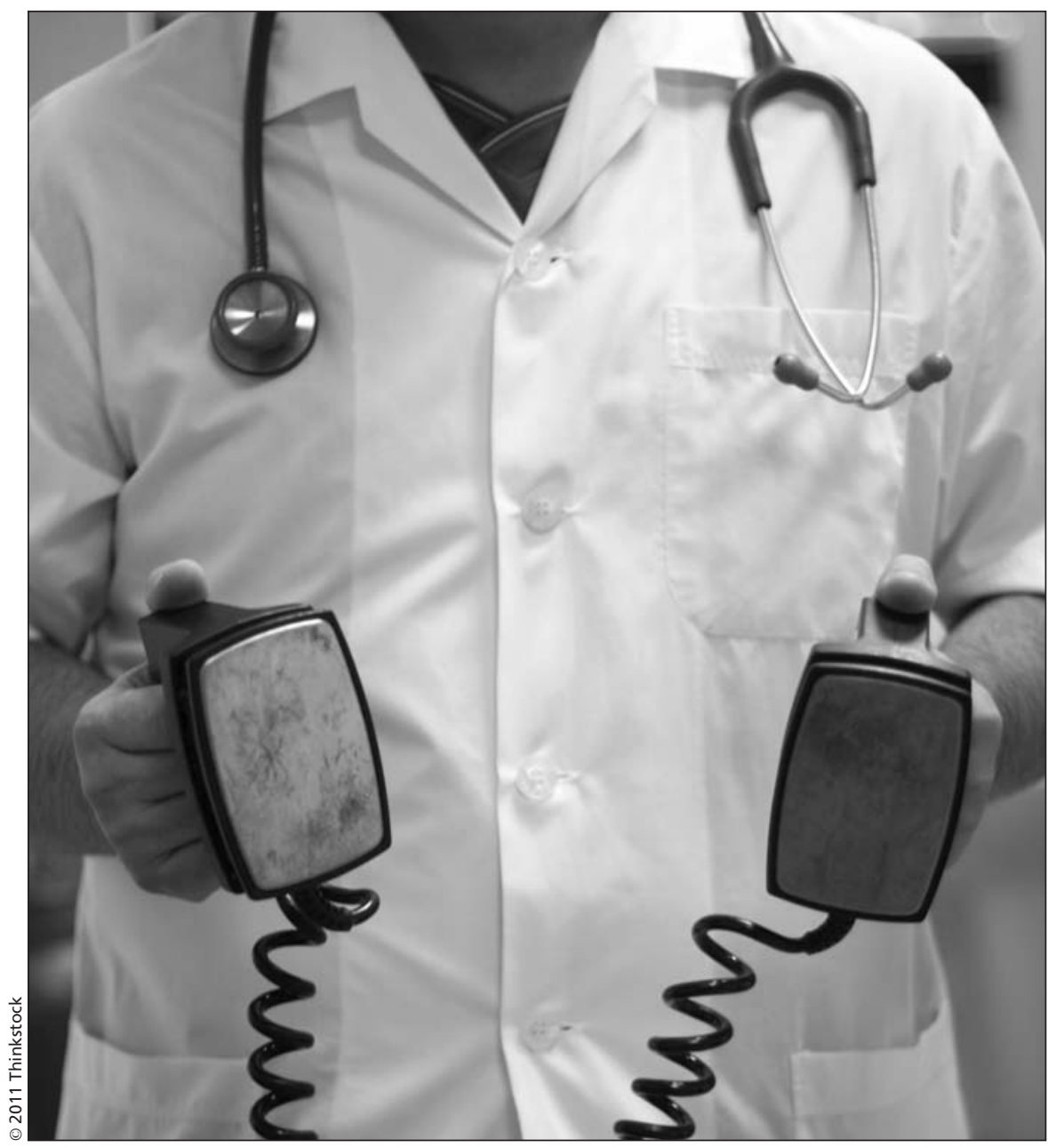

Whether or not to resuscitate can be a very tricky decision.

and developing ethical solutions for future resource shortages. "We recognize resources are finite, so the question becomes not if, but how we ration. Openly, transparently, democratically and with careful consideration for the rights of the vulnerable? Or quietly, without debate and behind closed doors?"

Health expenditures consume more than $50 \%$ of revenues in six of Canada's 10 provinces, not including federal transfers, and are on pace to consume some $75 \%$ of revenues in five provinces by the year 2019, according to the Fraser Institute (www.fraserinstitute.org/researchnews/research/display.aspx?id=10758).

Canada's aging population and the development of expensive therapies for life-threatening illnesses are escalating pressures on the health care system, particularly intensive care units. Surveys estimate that Ontario, for example, will need $80 \%-93 \%$ more intensive beds over the next 20 years, while $87 \%$ of intensive care physicians said they provided "futile" care at least once in the previous year (www.cmaj.ca/lookup/doi /10.1503/cmaj.070144).

"We have to start making some difficult rationalizations about who we're going to treat," argued a cardiac surgeon at a January town hall meeting on health care transformation hosted by the Canadian Medical Association (www.cmaj.ca /lookup/doi/10.1503/cmaj.109-3797).

“We don't say at 85 you've lived well 
and there are some criteria by which I'm going to do a double valve replacement on you, but we should judge whether or not the potential years of life saved can be justified by that treatment."

Many patients may not need hospitalization near end-of-life, when most costs accrue, and would be better served by such strictures, added panelist Dr. John Ross, provincial advisor on emergency care for Nova Scotia. "I know a number of quite demented people right now who are getting various types of tests done, dialysis and various types of interventions. When you look at where this effort is going, what the end point is, is that really a valuable way to spend that diagnostic testing and dying person's time?"

Some believe Canada already has a "scarcity model" of health care resource allocation, says Krista Flint, a disability rights activist from Calgary, Alberta. "It's evidenced in things like the exclusion criteria built into new provincial pandemic planning protocols. In the case of a flu pandemic, for example, having a developmental disability now excludes you from access to a ventilator." Such protocols set a precedent for withholding or withdrawing beneficial treatment from vulnerable groups, she argues.

In a similar vein, the government of Ontario is developing new critical care triage guidelines to clarify which patients should receive limited intensive care resources (www.health.gov.on.ca /english/providers/program/critical_care /cct_access.html).

Under Canadian law, doctors don't have to provide treatment they deem "futile," says Dr. Laura Hawryluck, the physician lead on the strategy and an assistant professor of medicine at the University Health Network in Toronto, Ontario. However, without clear consensus about what constitutes futility, clinicians have no clear standard for weighing medical versus nonmedical criteria in their decision-making, she adds.

Studies have found that bed availability, patient age, physician attitudes, threat of legal action and pressure from superiors and family members come into play in admissions of patients to intensive care (Crit Care 2006;10:242).

Moreover, nonmedical reasons for triage decisions are rarely discussed openly, while institutional discussions on appropriate use of resources and how to incorporate cultural and religious beliefs into decision-making remain vague.

Hawryluck proposes a working definition of futility: "the use of considerable resources without a reasonable hope that the patient would recover to a state of relative independence or be interactive with their environment."

But without consensus on what constitutes "considerable resources ... reasonable hope ... relative independence ... (or) interactivity" with one's environment, this can easily become a slippery slope to denying treatment to the elderly, the disabled or people with chronic diseases who might otherwise live longer and have a quality of life that they find acceptable, Farlow says.

"These are really existential questions about judging quality of life," says Rhonda Wiebe, cochair of the Council of Canadians with Disabilities' end-oflife ethics committee. "I worry we'll get to a place where we're the sum of our illnesses. I know that if there's one respirator and two people, one who will need it for 12 hours and the other who will need it for the rest of their life, there's a split second allocation decision that's going to be made, and likely in the favour of the healthier person."

Many physicians already struggle to balance patient responsibilities with the "intense pressure" to conserve resources, says Dr. Ellen Tsai, a pediatric interventionist at Kingston General Hospital in Ontario. "We don't like to admit that we're placing value on people's lives and I think many physicians would prefer not to have that decision on their shoulders at all. At the same time, as the people who are best able to determine the medical benefit of a treatment, I think it would be wrong of us to entirely abdicate that responsibility."

Physicians make $80 \%$ of resource allocation decisions at the bedside, putting them in the role of "double agents," says Margaret Sommerville, founding director of the McGill University Centre for Medicine, Ethics and Law in Montréal, Quebec. "There's a clear ethical rule that a doctor has a primary obligation of personal care for each patient, meaning a doctor cannot put the interests of a group, society or another patient in front of the best inter- ests of the patient he or she is treating."

Patients, families or surrogate decision-makers who disagree with physicians are "left with no other alternative than to go to court to get an injunction to prevent discontinuing treatment," though most patients die before their cases are heard, says Mark Handelman, a Toronto-based lawyer who specializes in disputes over end-of-life care. There are also considerable legal ambiguities surrounding end-of-life decision-making authority (www.cmaj.ca/lookup/doi /10.1503/cmaj.109-3910).

A solution to many of these thorny issues might be to remove physicians from the rationing process, Sommerville says. "At an institutional level, hospitals and health authorities have an obligation to take into account other people's requirements, rights and needs, and can put efficiency ahead of any one patient in ways a doctor cannot."

Potential criteria proposed for future rationing schemes at institutional, regional or provincial levels include limitations on treatments by age, life expectancy or a patient's personal responsibility in an illness, but "none of them are foolproof," says Tsai. "A lot of talk about health care rationing fails to focus on actual algorithms for implementation on the ground. We have difficulty saying you're a 73-year-old former smoker who now has end stage heart disease and your score is 52 and this is where you fit on the scale of resources we'll provide."

The public is also unlikely to agree on which services are fair game for exclusion, judging from past attempts to transparently rationalize care in the United States, England, New Zealand and the Netherlands (CMAJ 2001;164[11]:158387). The state of Oregon's experience suggests that the more transparent a decision to ration care, the more difficult it is to implement because public opposition invariably emerges.

"No one wants to be seen as the person that started the conversation on who we exclude from universal health care," Farlow says. "But we're already excluding people like my daughter in other subtle ways, so the time to talk is now." Lauren Vogel, CMAJ

CMAJ 2011. DOI:10.1503/cmaj.109-3932 